\title{
Non-equilibrium Thermodynamics, Thermomechanics, Geodynamics
}

\author{
Klaus Regenauer-Lieb ${ }^{1,2}$, Bruce Hobbs ${ }^{1}$, Alison Ord ${ }^{1}$, and Dave A. Yuen ${ }^{3}$ \\ ${ }^{1}$ CSIRO Exploration and Mining, PO Box 1130, Bentley, WA 6102, Australia \\ ${ }^{2}$ School of Earth and Geographical Sciences, The University of Western Australia, Perth, WA \\ 6009, Australia \\ ${ }^{3}$ Dept. of Geology and Geophysics and Minnesota Supercomputing Institute, Univ. of \\ Minnesota, Minneapolis, MN 55455-0219, U.S.A \\ \{Alison. Ord, Bruce.Hobbs, klaus.regenauer-lieb\} @csiro.au, \\ daveyuen@gmail.com
}

\begin{abstract}
The subject of non-equilibrium thermodynamics is now quite old, dating back to Prigogine, Biot and Truesdell in the 1950's and 1960's. It has had a resurgence in the physical sciences in the past five years largely due to a consolidation of ideas on thermodynamics as a whole and to advances in computer speeds. Non-equilibrium thermodynamics has now advanced to a stage where it forms an umbrella approach to understanding and modelling coupled phenomena in Earth Sciences. Currently, approaches are pioneered independently in geodynamics, seismology, material sciences (solid mechanics), atmospheric and marine sciences (fluid dynamics) and the chemical sciences. We present a first attempt at consolidating some ideas and show a simple example with potential significance for geodynamics, structural geology and seismology.
\end{abstract}

Keywords: Thermodynamics, Geodynamics, Structural Geology, Slow Earthquakes.

\section{Introduction}

In this contribution we advance new approaches for the problem of deformation of geological solids and attempt to develop a common framework for the different approaches [1-4]. The application of thermodynamics to the plastic deformation of solids requires special caution in a number of respects [5]. First, plastic deformation is essentially irreversible. It is hence important to define a reference state from which deviations define a driving force. Second, plastic deformation depends sensitively on shear stresses, and it leads to shape changes and not just volume changes. While volumetric strain is easily defined as a state variable, the definition of a nonvolumetric strain as a state variable is not straight forward. Third, in plasticity, the number of relevant material parameters may far exceed the number of macroscopically observable relations; the use of energy, volume, etc., as "state variables" then may become meaningless. 
In the light of these difficulties it is obvious that thermodynamic approaches to continuum mechanics have, in the past, been replaced by constitutive approaches. These have up to now neglected the basic principles of thermodynamics. However, recently, significant progress has been made for understanding the plastic deformation of soils where all three cautions can be addressed rigorously [6]. Continuum mechanics can be recast and verified on the basis of thermodynamics for isothermal deformation. For soils the influence of the state variable temperature, is thought to be negligible. It is hence possible, to formulate, to first order, a theory of plastic deformation without local gradients in temperature, which must arise naturally through the process of dissipation.

\subsection{Isothermal Thermomechanics}

Thermomechanics has been pushed forward as a robust theory in the 70s [7] continuing earlier breakthroughs in thermodynamics of chemical systems. However, since then the topic of thermomechanics of continua was not considered forefront of continuum mechanics. Recently, thermomechanics has been rediscovered [8]. Modern thermomechanical approaches assume that there is no heat flow, thus allowing a very concise treatment of the basic underlying principles. Thermomechanics addresses only the second law of thermodynamics as an overarching constraint to deformation. This solves the reference state for irreversible deformation by constraints on energy.

The strain energy rate density is defined as the double dot product (scalar product) of the applied stress $\boldsymbol{\sigma}$ and the strain rate tensor thus the local power (work rate) of a reference volume [9].

$$
\boldsymbol{\sigma}: \dot{\boldsymbol{\varepsilon}}=\dot{\Psi}+\Phi
$$

where $\Psi$ is the Helmholtz free energy function and $\Phi$ is the rate of dissipation per unit volume. In the isothermal case the Clausius-Duhem Inequality (second law of thermodynamics) collapses to:

$$
\Phi \geq 0
$$

The Clausius-Duhem Inequality can hence be understood as the isothermal thermalmechanical stability condition. Note, that the Clausius-Duhem Inequality has a broader meaning in that not only the mechanical dissipation is positive but that the thermal conduction is positive. Combining eqns. (1) and (2) it follows that the thermomechanical equilibrium state is constrained by the condition that the rate of change of the Helmholtz free energy is smaller than the local strain energy rate density [5].

$$
\dot{\Psi} \leq \boldsymbol{\sigma}: \dot{\boldsymbol{\varepsilon}}
$$

The equality holds for isentropic (elastic) deformation while plastic deformation causes dissipation.

The second caution of a definition of a strain measure as a differential function of the free energy is solved by expanding the local strain rate energy density into dissipative and non-dissipative microstrains $\alpha_{\mathrm{k}}$ assuming a summation over $k$ of the various microstructural processes 


$$
\boldsymbol{\sigma}: \dot{\boldsymbol{\varepsilon}}=\frac{\partial \Psi}{\partial \boldsymbol{\varepsilon}}: \dot{\boldsymbol{\varepsilon}}+\frac{\partial \Psi}{\partial \boldsymbol{\alpha}^{k}}: \dot{\boldsymbol{\alpha}}^{k}+\frac{\partial \Phi}{\partial \dot{\boldsymbol{\alpha}}^{k}}: \dot{\boldsymbol{\alpha}}^{k}
$$

In the same framework the Cauchy stress tensor can be defined as a partial derivative of the Helmholtz free energy.

$$
\boldsymbol{\sigma} \equiv \frac{\partial \Psi}{\partial \varepsilon}
$$

and the small strain tensor as the partial derivative of the Gibbs free energy:

$$
\varepsilon \equiv \frac{\partial G}{\partial \boldsymbol{\sigma}}
$$

The partial derivatives of the Helmholtz free energy (equation 4) and the dissipation potential over their microstrains and microstrain rates, respectively, define the recoverable elastic small strain measure and the dissipative small strain measure, thus giving the familiar additive elasto-dissipative strain rate decomposition [10].

$$
\dot{\boldsymbol{\varepsilon}}=\frac{\partial^{2} G}{\partial \boldsymbol{\sigma}^{2}}: \dot{\boldsymbol{\sigma}}+\frac{\partial^{2} G}{\partial \boldsymbol{\sigma} \partial \boldsymbol{\alpha}^{k}}: \dot{\boldsymbol{\alpha}}^{k}=\dot{\boldsymbol{\varepsilon}}^{\text {elastic }}+\dot{\boldsymbol{\varepsilon}}^{\text {diss }}
$$

The third caution put forward by Kocks et al. [5] is the caution that the number of necessary material parameters for interpreting the strongly nonlinear system can exceed the number of available equations. For isothermal approximations (e.g. soil) this does not appear to be the case [6]. For the more general application to nonisothermal cases it is a challenge to modern computational approaches. Nowadays, such complex non-linear systems can readily be analyzed by computational methods.

\subsection{Non-isothermal Thermomechanics}

Temperature needs to be added as a state-variable, therefore we have to consider a full thermodynamic approach where heat $Q$ is allowed to flow into the reference volume. Integrating the strain energy rate density in equation (1) with respect to time, we obtain the mechanical work $W$ done in the volume element. The first law of thermodynamics states that in a closed system the increment of specific internal energy $\delta U$ of the reference volume is the sum of the increment of heat $\delta Q$ flowing into the solid and the increment of mechanical work $\delta W$ done on the solid.

$$
\delta U=\delta Q+\delta W .
$$

The internal energy is also defined by:

$$
U \equiv \Psi\left(T, \alpha_{j}\right)+s T,
$$


where the internal energy is now written in terms of entropy $S$ and $\alpha_{j}$ are state variables other than temperature $T$. The entropy is defined by:

$$
s \equiv-\frac{\partial \Psi}{\partial T}
$$

The first law is often expressed in irreversible thermodynamics in terms of the equivalent time derivatives rather than using virtual variations. Taking the material time derivative (in our notation $D / D t$ ) of the entropy we obtain

$$
\frac{D s}{D t}=-\frac{\partial^{2} \Psi}{\partial T^{2}} \frac{D T}{D t}-\frac{\partial^{2} \Psi}{\partial T \partial \alpha_{j}} \frac{D \alpha_{j}}{D t}
$$

where the specific heat $c_{\alpha}$ is defined as:

$$
c_{\alpha} \equiv-T \frac{\partial^{2} \Psi}{\partial T^{2}}
$$

We switch from a unit volume based to a unit mass based framework ( $\tilde{\Psi}=\Psi / \rho)$, the entropy flux is then related to the heat by:

$$
\rho T \frac{D s}{D t}=\rho c_{\alpha} \frac{D T}{D t}-\rho T \frac{\partial^{2} \tilde{\Psi}}{\partial T \partial \alpha_{j}} \frac{D \alpha_{j}}{D t} .
$$

Considering equations (8-10) and rearranging terms we can now write the heat equation as [11].

$$
\rho c_{\alpha} \frac{D T}{D t}=\left(\boldsymbol{\sigma}: \dot{\boldsymbol{\varepsilon}}-\rho \frac{\partial \tilde{\Psi}}{\partial \alpha_{j}} \frac{D \alpha_{j}}{D t}\right)+\rho T \frac{\partial^{2} \tilde{\Psi}}{\partial T \partial \alpha_{j}} \frac{D \alpha_{j}}{D t}+q_{i},
$$

where the first term described by the bracket on the right side is the mechanical dissipation potential $\Phi$ defined in equation (1), the second term is the isentropic thermal-mechanical coupling term and $q_{i}$ is the heat flux.

This equation is a generalized thermodynamic formulation without specification of the mode of mechanical dissipative and stored microstrain-processes, as well as the particular mechanism heat is transferred. It describes the importance of two important thermal-mechanical feedback effects within the energy equation. The first bracketed term on the right side, the dissipated energy, is also known as the shear heating term, and the second term is the important feedback given by the stored energy. While this equation is generally applicable we will now go on and illustrate the application of the method in a simple example for a specific case study, simplifying what we believe to be the most essential case in geodynamics. 


\subsection{Simplified Thermodynamic Approach for Geodynamics}

In terms of non-dissipative processes producing elastic strain we only consider thermal expansion and elastic deformation:

$$
\dot{\boldsymbol{\varepsilon}}^{\text {elastic }}=\left(\frac{1+v}{E} \frac{D \tilde{\sigma}^{\prime}}{D t}+\frac{v}{E} \frac{D p}{D t}+\alpha \frac{D T}{D t} \delta_{i j}\right) .
$$

where $E$ is Young's modulus, $v$ is Poisson ratio and and $\alpha$ is the coefficient of thermal expansion. $\tilde{\sigma}^{\prime}$ is the objective co-rotational stress tensor and $\delta_{i j}$ is the Kronecker delta. For the dissipative processes in equation (7) we assume only two basic micro-mechanisms causing macroscopic dissipative strains:

$$
\dot{\boldsymbol{\varepsilon}}^{\text {diss }}=\left(\dot{\boldsymbol{\varepsilon}}^{p l} \frac{\sigma^{\prime}}{2 \tau}\right)_{\text {plastic }}+\left(A \sigma^{\prime} J_{2}^{n-1} \exp \left(-\frac{Q}{R T}\right)\right)_{\text {creep }} .
$$

The plastic yield stress is $\tau$ and is here assumed to be linearly dependent on pressure; $A$ and $n$ are power-law material constants, $Q$ is the activation enthalpy and $R$ is the universal gas constant. $J_{2}$ is second invariant of the deviatoric stress tensor $\sigma^{\prime}$.

The crucial step for a computational approach producing patterns out of nonlinear thermodynamic feedback processes is common to all ab-initio style calculations. Here, the method is applied to homogenized microstrain processes in a continuum approach upscaled for geodynamics. The key is to solve the triplet of equilibrium equations, continuity, momentum and heat in a fully coupled way. Without coupling there is no pattern development. The computational strategy, the convergence requirements, and the time stepping routine are laid out in different paper [12]. Here we will just point out the two key feedback loops appearing in the form of the energy equation (14) which now reads:

$$
\rho c_{P} \frac{D T}{D t}=\boldsymbol{\sigma}: \dot{\boldsymbol{\varepsilon}}^{\text {diss }}+\alpha T \frac{D p}{D t}+\rho c_{P} \kappa \nabla^{2} T .
$$

Discussing this specific simplification of equation (13) from back to front we now only consider for $q_{i}$ the phonon part of heat conduction with thermal diffusivity $\kappa$, $c_{P}$ is the specific heat at constant pressure. For the isentropic feedback term we only consider the state variable pressure $p$ including the thermal-elastic feedback loop and in the first term on the right we consider that all dissipated work is released as heat. This is of course an extreme mathematical idealization of a real material. For most materials the shear heating efficiency appears to be around $85 \%$ and $95 \%$ for large strain [13]. The remaining 5-15\% clearly require incorporation of additional feedback loops in the expansion of the second term of equation (14), thereby diminishing the energy available for shear heating feedback by the same amount $[1,3]$. This feedback is important for very shallow crustal conditions. We only described deeper crustal conditions. A surprising dynamics, not recorded in classical quasi-static continuum mechanical approaches, is based on the physics of two feedback loops. 


\section{Feedback Loops}

With this formulation we have encapsulated two basic thermodynamics feedback processes into the energy equation, based on the two state variables temperature and pressure, these are: 1) Thermal expansion feedback dependent on elasto-plastic (semi-brittle, $p$-dependent) displacements; 2) Shear heating feedback dependent on elasto-visco-plastic (ductile, $T$-dependent) displacements [14].

\section{Model}

The model setup is a very basic one. We consider an isothermal quartz-feldspar composite slab with random thermal perturbations and extend it by a factor of 4 from $3.3 \times 12 \mathrm{~km}$ to $13.2 \times 3 \mathrm{~km}$. This is considered to be a generic slice of a continental crust, where the individual $p-T$ conditions correspond to a particular depth. Since the pressure and temperature increases in the crust with depth, the pressure sensitive brittle feedback mechanism is suppressed for higher starting model temperatures. Random temperature perturbations with a maximum amplitude of $70 \mathrm{~K}$ are assumed at the start of the extension and they diffuse during deformation and self-organize into a characteristic pattern controlled by the two feedback loops. Owing to the pure shear boundary conditions, the feldspar and quartz layers are expected to assume a shape shown in Figure 1, either through plastic deformation or viscous creep or both, depending on the base temperature/pressure assumed at the start of the model run. No internal structure is expected in the absence of thermal-mechanical feedback.

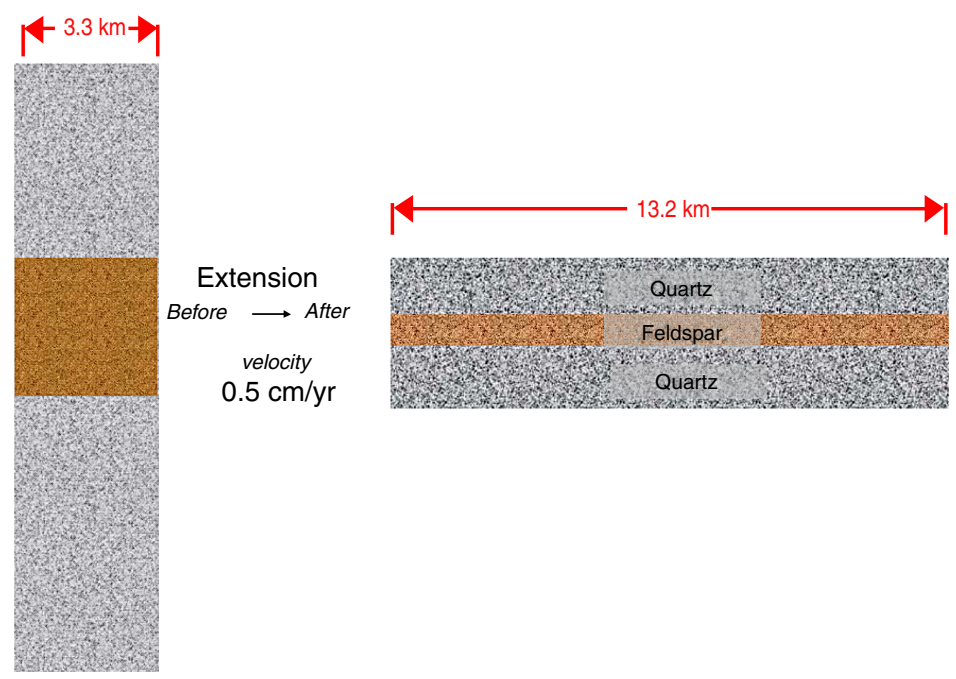

Fig. 1. Pure shear extension of an isothermal composite piece of continental crust. Without thermal-mechanical feedback random thermal perturbation have no effect and both feldspar and quartz are extended homogenously as shown. 


\section{Results}

The resulting patterns deviate significantly from the expected patterns for a standard continuum mechanics solution. Figure 2 clearly shows three significantly different mechanical regimes.

We have shown here an example of the new approach originally designed for geodynamic simulations [14]. The rich spatial patterns developing out of an originally unpatterned state are typical for thermodynamic feedback. The $500 \mathrm{~K}$ case, in particular, is accompanied by a dynamic pattern of creep/plastic slip events, which could throw a light on the dynamic events underlying the source region of major earthquakes such as the recent Sumatra event [15].

430 Kelvin Extension

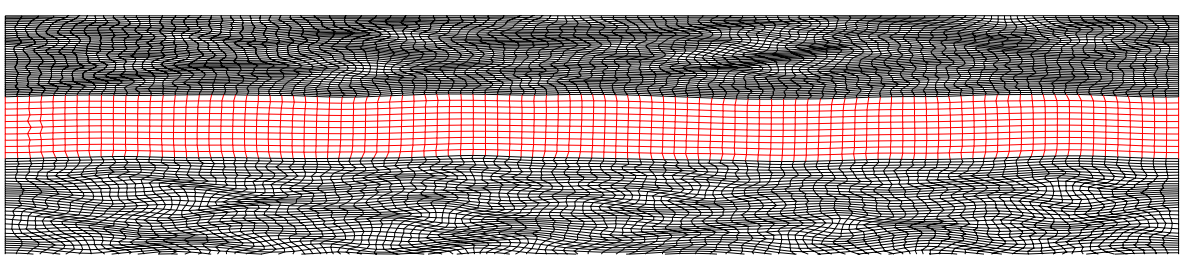

500 Kelvin Extension

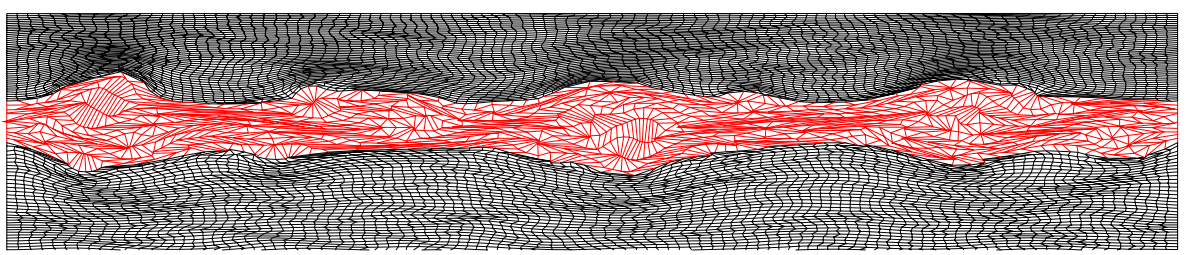

530 Kelvin Extension

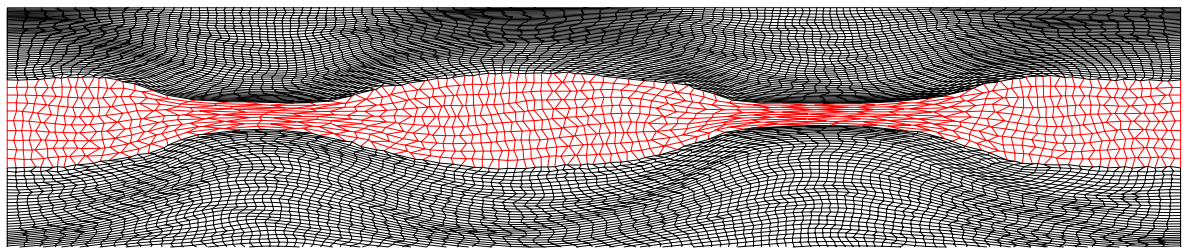

Fig. 2. Only the final pattern after extension is shown. With feedback random temperature perturbation have a significant effect on the deformation. Three mechanical regimes are identified. At $430 \mathrm{~K}$ brittle, thermal-expansion feedback dominates, at $530 \mathrm{~K}$ ductile shearheating feedback dominates. Mixed-mode brittle/ductile feedback produces the richest pattern at around $500 \mathrm{~K}$.

\section{Summary and Outlook}

Computer sciences are now allowing us to tackle problems that have previously been out of reach of exact analytical approaches. This revolutionizes new non-equilibrium 
thermodynamic approaches for coupling chemistry and mechanics (both fluid and solid). We have shown here a promising research path with the potential to bridge seismology, geodynamics, geomechanics and materials sciences in the near future.

Acknowledgments. $P m d^{*} C R C$, CSIRO, UWA , DAY acknowledges support from ITR and CSEDI programs of National Science Foundation.

\section{References}

1. Y. Ben-Zion, V. Lyakhovsky: Analysis of Aftershocks in a Lithospheric Model with Seismogenic Zone Governed by Damage Rheology, Geophysical Journal International 165, 197 (2006).

2. I. F. Collins: The concept of stored plastic work or frozen elastic energy in soil mechnics, Geotechnique 55, 373 (2005).

3. D. Bercovici, Y. Ricard: Energetics of a two-phase model of lithospheric damage, shear localization and plate-boundary formation, Geophysical Journal International 152, 581 (2003).

4. B. Hobbs, K. Regenauer-Lieb, A. Ord: The thermodynamics of folding in the middle to lower crust, Geology 35, 175 (2007).

5. U. F. Kocks, A. S. Argon, M. F. Ashby, Thermodynamics and kinetics of slip (Pergamon Press, Oxford, 1975), pp. 293.

6. I. F. Collins: Elastic/plastic models for soils and sands, International Journal of Mechanical Sciences 47, 493 (2005).

7. H. Ziegler, An Introducton to thermomechanics (North-Holland Publishing Company, Amsterdam, ed. 2nd Edition, 1983), pp. 358.

8. I. F. Collins, G. T. Houlsby: Application of thermomechanical principles to the modelling of geotechnical materials, Proceedings - Royal Society of London, A 453 1964, 1975 (1997).

9. I. F. Collins, P. A. Kelly: A thermomechanical analysis of a family of soil models, Geotechnique 52, 507 (2002).

10. S. Nemat-Nasser: Decomposition of strain measures and their rates in finite deformation elasto-plasticity, International Journal of Solids and Structures 15, 155 (1979).

11. A. Chrysochoos, R. Peyroux: Modelisation numerique des couplages en thermomecanique des solides, Revue europeene des element finis 6, 673 (1997).

12. K. Regenauer-Lieb, D. A. Yuen: Positive feedback of interacting ductile faults from coupling of equation of state, rheology and thermal-mechanics, Physics of Earth and Planetary Interiors 142, 113 (2004).

13. A. Chrysochoos, O. Maisonneuve, G. Martin, H. Caumon, J. C. Chezeaux: Plastic and dissipated work and stored energy, Nuclear Engineering and Design 114, 323 (1989).

14. K. Regenauer-Lieb, R. Weinberg, G. Rosenbaum: The effect of energy feedbacks on continental strength, Nature 442, 67 (2006).

15. K. Regenauer-Lieb, D. Yuen: Quartz Rheology and short time-scale crustal instabilities, Pure and Applied Geophysics 163 (1915-1932, 2006). 